\title{
ReLAInEP
}

\section{TESTE DE CONSUMIDORES E ANÁLISE DE APARÊNCIA, SABORES E CORES PARA O DESENVOLVIMENTO DE NOVOS PRODUTOS: O CASE DO PROJETO DE BROINHAS DE MILHO SABOREADAS}

\author{
Fernando Henrique Lermen ${ }^{1}$ \\ Gustavo de Souza Matias ${ }^{2}$ \\ Flávia Açafrão Modesto ${ }^{3}$ \\ Rodrigo Röder ${ }^{4}$ \\ Thays J. Perassoli Boiko $\mathbf{r}^{5}$
}

\begin{abstract}
RESUMO: O Do ponto de vista de mercado, um produto é aquilo que o consumidor percebe como capaz de satisfazer uma necessidade material. Assim, a noção de novo varia conforme o critério que se utiliza na diferenciação do produto em relação aos seus concorrentes, pois, mesmo sem que haja algo técnico e objetivamente novo o produto pode ser percebido como tal pelo consumidor. Neste artigo, a análise do Desenvolvimento de Novos Produtos é voltada para a área alimentícia, onde o produto analisado inclui-se na área dos congelados, sendo ele Broinhas de Milho Saboreadas. Para o desenvolvimento deste, manteve-se o foco em alguns requisitos básicos, como: cor; sabor, e; aparência. O método de procedimento foi o hipotéticodedutivo. A revisão de literatura foi realizada no nos portais Scielo e Capes. Para analisa-lo, foi feito o Teste de Consumidores. Neste teste, o produto foi degustado e depois avaliado através de um questionário. Diante disso, pode-se observar que o ponto forte das Broinhas de Milho Saboreadas são a aparência e o sabor, onde a cor deve ser aprimorada. Como pesquisas futuras aconselha-se a aplicação desta análise para outros desenvolvimentos de novos produtos alimentícios.
\end{abstract}

Palavras-chave: Projeto do Produto; Atributos de Produtos; Produtos Congelados; Teste com Consumidores.

\footnotetext{
1 Universidade Estadual do Paraná - Campus de Campo Mourão (UNESPAR/CM) (fernandolermen@hotmail.com)

${ }^{2}$ UNESPAR/CM (gusmatias@ gmail.com)

3 UNESPAR/CM (fla_vinha22@hotmail.com)

${ }^{4}$ UNESPAR/CM (rodrigoroder@hotmail.com).

5 UNESPAR/CM e Universidade de São Paulo - Escola de Engenharia de São Carlos (thaysperassoli@bol.com.br).
}

ReLAInEP - Revista Latino-America de Inovação e Engenharia de Produção, Curitiba, PR, Brasil, v. 3, n. 4, p. 97-109, 2015. 


\section{ReLAInEP}

ABSTRACT: From the perspective of market, a product is what the consumer perceives as able to satisfy a material necessity. Thus, the notion again varies according to the criterion that is used on product differentiation relative to its competitors, because even without something technical and new product can objectively be perceived as such by the consumer. In this article, the analysis of New Product Development is focused on the food industry, where the analyte is included in the area of frozen, being scones Corn Enjoyed. To develop this, the focus remained on some basic requirements such as color, flavor and appearance. The method of procedure was the hypothetical-deductive. A literature review was performed in Scielo and Capes Journals Portals. To analyze it, the test was done Consumers. In this test, the product was sampled and then assessed by a questionnaire. Thus, it can be seen that the strong point of the Corn Enjoyed scones are the appearance and flavor, where the color should be improved. As future research we recommend the application of this analysis to other developments of new food products.

Keywords: Product Project; Attributes of products; Frozen products; Testing with Consumers.

RESUMÉN: Desde el punto de vista del mercado, el producto es lo que percibe el consumidor como capaz de satisfacer una necesidad física. Por lo tanto, la noción de nuevo varía de acuerdo con los criterios que se utiliza para diferenciar el producto en relación a sus competidores, porque incluso sin algo técnica y objetivamente el nuevo producto puede ser percibido como tal por el consumidor. En este artículo, el análisis del desarrollo de nuevos productos se centra en la industria alimentaria, en el producto de analice está incluido en el área de congelados, siendo disfrutado bollos de maíz. Para desarrollar este, se mantuvo enfocado en algunos requisitos básicos, tales como: el color; sabor, y; apariencia. El procedimiento fue el hipotético-deductivo. La revisión de la literatura se llevó a cabo en los portales Scielo y Capes. Para analizarlo fue hecha una evaluación con los Consumidores. En esta prueba, el producto se muestreó y luego evaluada a través de un cuestionario. Así, puede verse que la fuerza de los bollos de maíz Disfrutamos son el aspecto y el sabor, donde el color debe ser mejorado. Como aplicación de la investigación futura se aconseja de este análisis a otros desarrollos de nuevos productos alimenticios.

Palabras clave: El diseño del producto; Características del producto; Los productos congelados; Los consumidores prueban.

\section{INTRODUÇÃO}

Produto é tudo aquilo que pode ser oferecido a um mercado para apreciação, aquisição, uso ou consumo e para satisfazer um desejo ou uma necessidade do consumidor. É também um conjunto de atributos tangíveis e intangíveis que proporciona benefícios reais ou percebidos (SEMENICK E BAMOSSY, 1995, p. 260).

O Project Managmente Institute (PMI) (1996) define projeto como um esforço temporário, levando a efeito para criar um produto/serviço único. Promon (2008) definiu projeto como sendo um empreendimento temporário realizado de forma progressiva. Por

ReLAInEP - Revista Latino-America de Inovação e Engenharia de Produção, Curitiba, PR, Brasil, v. 3, n. 4, p. 97-109, 2015. 
serem temporários, os projetos têm, obrigatoriamente, início e término definidos, diferenciando-se de operações contínuas, onde é essa característica a qual não indica, necessariamente, que sejam curtos ou longos, mas apenas que são iniciados, evoluem e, por fim, são finalizados (PROMON, 2008).

Além de definir produto e projeto, se faz necessário conceituar Projeto do Produto (PP), pois é onde se enquadram as atividades utilizadas no estudo de caso que está sendo apresentado. PP é "o processo de negócio cujas tarefas são: a) analisar as necessidades e expectativas dos stakeholders e criar uma especificação para o produto ou serviço, e; transmitir todas as informações sobre o projeto e o produto para todas as áreas funcionais da empresa" (MOREIRA, 2000 e GRUPO DE ENGENHARIA INTEGRADA - NUMA, s.d).

Baxter (2000) relata que a atratividade exercida pelos produtos pode ser classificada em quatro níveis: i) conhecimento prévio do produto, onde produtos já familiares aos consumidores eles pretendem continuar comprando; ii) atração semântica, onde o produto transmite a imagem de um bom funcionamento; iii) atração simbólica, onde o produto representa valores pessoais ou sociais do consumidor; iv) atração intrínseca, onde a forma do produto apresenta uma beleza própria.

O objetivo do presente trabalho é apresentar a análise de Aparência, Sabores e Cores para o Desenvolvimento de Novos Produtos, realizada no case do Projeto de Broinhas de Milho Saboreadas. O Projeto foi desenvolvido, em 2012, como um uma exigência de uma Disciplina de Projeto de Produto (LERMEN et al., 2012).

Os resultados do Teste com Consumidores, utilizado nesta análise, são apresentados.

O tema do presente artigo se enquadra nas Áreas e Subáreas de Conhecimento de Engenharia de Produção, apresentadas pela Associação Brasileira de Engenharia de Produção (ABEPRO) (2008) na área de Engenharia do Produto e subárea Gestão de Desenvolvimento do Produto.

$\mathrm{O}$ artigo está estruturado em seis seções. Na primeira o assunto é ambientalizado e objetivo do trabalho é apresentado. Na seção seguinte, apresenta-se a fundamentação teórica sobre a importância da Aparência, Cor e Sabor no Desenvolvimento de Novos Produtos e sobre os Aspectos do Desenvolvimento de Produtos Semiprontos ou de Rápido Preparo. Na terceira seção apresenta-se a Revisão de Literatura realizada. Em seguida, está a Metodologia

ReLAInEP - Revista Latino-America de Inovação e Engenharia de Produção, Curitiba, PR, Brasil, v. 3, n. 4, p. 97-109, 2015. 


\section{ReLAInEP}

de Pesquisa utilizada. Na quinta seção, apresentam-se os resultados deste trabalho. Por fim, estão as Considerações Finais.

\section{FUNDAMENTAÇÃO TEÓRICA}

\subsection{Importâncias da Aparência no Desenvolvimento de Novos Produtos}

Quando falamos de um produto atrativo, segundo Baxter (2000), raramente nos referimos ao seu som, cheiro ou paladar, mais sim ao estilo visual, pois o sentido visual é predominante sobre os demais sentidos, onde a atratividade de um produto depende então do aspecto visual.

Conforme Cobucci (2010), a aparência é o primeiro dos atributos sensoriais observados em um produto. A aceitação do produto pelo consumidor é dependente deste aspecto, pois, a aparência é responsável pelas primeiras impressões sobre o produto, trazendo informações sobre as outras características como cor, forma e tamanho, viscosidade, consistência de líquidos, textura da superfície e brilho.

De acordo com Penna (1999) apud Barbosa, Freitas e Waszynskyj (2003), os alimentos além de seu valor nutritivo devem agradar e satisfazer ao consumidor através do equilíbrio das diversas características sensoriais. Ao desenvolver um novo produto alimentício é fundamental aperfeiçoar características como forma, textura, cor, odor, aparência e a interação dos principais componentes, almejando o alcance de um equilíbrio entre essas características que resulte em alta qualidade e por consequência aceitabilidade do produto no mercado.

Para que um produto desconhecido seja consumido é necessário que este atraia o consumidor, porém é essencial que o produto tenha uma boa aparência para que seja consumido.

\subsection{Importâncias da Cor no Desenvolvimento de Novos Produtos}

Um dos aspectos principais para a aceitação de um produto são as cores, responsáveis por $60 \%$ da aceitação deste. O efeito das cores pode influenciar outras emoções e sentimentos, podendo fazer o consumidor sentir-se mal ou sentir-se bem, atraí-lo ou distraí-lo, estimular ou não o seu apetite (PETTER \& OLSON, 2009).

Segundo Battistella, Colombo e Abreu (2010), as cores são responsáveis por estimular e direcionar para o público que o produto é destinado, sendo ligada ao físico e imaginada em

ReLAInEP - Revista Latino-America de Inovação e Engenharia de Produção, Curitiba, PR, Brasil, v. 3, n. 4, p. 97-109, 2015. 
pensamento. A cor pode ser usada para atrair ou repelir a compra de um produto, estando ligado a fatores culturais. Cada consumidor tem preferência por determinado tipo de cor para cada tipo especifico de produto.

Para Battistella, Colombo \& Abreu (2010), a reação que os consumidores têm sobre as cores é puramente emocional: cores fortes como vermelho tem uma reação imediata e impactante; algumas cores podem ser usadas para dar a impressão de um produto ser barato ou caro, como é caso do dourado que dá um aspecto de artigo de luxo ao produto; cores forte podem ser usadas para indicar a qualidade de produtos alimentícios; cores como vermelho e laranja tem alta percepção.

Para Petter \& Olson (2009), o amarelo é a cor que o cérebro percebe mais rapidamente.

Segundo Cobucci (2010), a cor é um dos atributos de maior impacto na avaliação do consumidor sobre um produto, o consumidor espera que os produtos tenham uma cor característica, evitando produtos cuja cor não esteja de acordo com o esperado. Os consumidores costumam associar a cor dos produtos a outras características, um exemplo disso é a laranja, pois há uma crença entre os eles de que sua cor está associada ao sabor, estes acreditam que quanto mais amarela for à casca da laranja mais doce será a sua polpa, daí temse a importância de oferecer uma cor características para os produtos em desenvolvimento, pois essa será fundamental para sua aceitação.

A cor de um produto é um aspecto que o consumidor relaciona com a própria qualidade deste, apesar de muitos fatores psicológicos estarem envolvidos no processo, para aceitação de um novo produto a cor deve ter características que demonstram a sanidade do produto.

\subsection{Importâncias do Sabor no Desenvolvimento de Novos Produtos}

O sabor é um conjunto de sensações resultantes de estímulos químicos causados pela presença de alimento na cavidade bucal, essas sensações podem ser odores e gostos, odor e gosto são fatores responsáveis por estimular o consumo de produtos alimentícios (Cobbuci, 2010).

Para Cobbuci (2010), o odor é a sensação percebida ao se estimular o sentido do olfato, ou seja, é o atributo que pode ser percebido pelos receptores olfativos ao cheirar-se uma substancia volátil. Já gosto é uma sensação percebida na cavidade bucal e na língua através do 


\section{ReLAInEP}

sentido do gosto, existindo cinco gostos primários, ácido, salgado, doce, amargo e umami, o restante das sensações gustativas são provocadas por combinações destes 5 gostos.

Segundo Canabrava et al. (2006), o odor e o gosto estão relacionados e só é possível sentir o sabor dos alimentos através dos dois sentidos, olfato e paladar. Os receptores gustativos são ativados por substancias presentes nos alimentos e os receptores olfativos são ativados por substancias presentes no ar, mas muito do que as pessoas costumam chamar de gosto é resultado do olfato, pois ao quebrar determinados alimentos na mastigação o odor se espalha pelo nariz.

Para Cobbuci (2010), o odor é uma das características de um produto que possuem capacidade atrair ou repelir os consumidores, tendo grande importância na aceitação dos produtos alimentícios. O odor pode indicar para o consumidor a qualidade e a sanidade do produto.

Segundo Guyton (2006), Baptista, (2007) e Institute of Physics (2008) apud Strapasson et al. (2011), a percepção do sabor é um processo que permite que a pessoa escolha alimentos de acordo com suas preferências. Este processo é seletivo e subjetivo, a subjetividade da percepção do sabor refere-se a realidade individual criada pelo consumidor perante o produto, podendo ocorrer um desacordo entre o estimulo emitido pelo ambiente e o percebido pelo consumidor. A percepção dos aspectos de um alimento é diferente para cada consumidor e dependente da interação dos atributos sensoriais do produto com os aspectos psicológico do consumidor, pode-se tomar como exemplo disso o fato de os alimentos condimentados ou ácidos terem maior aceitação pelo público adulto que infantil.

Através dessas considerações, feitas sobre o sabor, nota-se que deve haver um equilíbrio entre o gosto e odor dos produtos. As características odoríferas e gustativas do produto devem ser projetadas para agradar o público a o qual o produto é destinado, tendo em vista que a aceitação deste dependerá do sabor e das características psicológicas do consumidor.

\subsection{Aspectos do Desenvolvimento de Produtos Semiprontos ou de Rápido Preparo}

O consumo das pessoas que moram sozinhas, segundo Ventura (2010), vem crescendo em média $6 \%$ ao ano, sendo este grupo de consumidores responsável por $40 \%$ do aumento das vendas de produtos práticos e em porções individuais. O crescimento de 10,6\%, entre 2006 e

ReLAInEP - Revista Latino-America de Inovação e Engenharia de Produção, Curitiba, PR, Brasil, v. 3, n. 4, p. 97-109, 2015. 


\section{ReLAInEP}

2007, dos alimentos congelados, o que representou mais do que o dobro do crescimento do setor alimentício.

Segundo Camargo, Queiroz e Sclaco (2007), nos debates sobre a segurança do alimento, destaca-se a existência de uma clara tendência dos consumidores procurarem produtos com maior qualidade, devido a preocupação com saúde, nutrição e segurança do alimento, aliado a conveniência de diferentes tipos de preparo rápido e produtos Semiprontos.

Matistella e Milan (2009) relatam que, as empresas atualmente visualizam um grande mercado para produtos semiprontos e de rápido preparo, onde apresenta uma grande necessidade dos consumidores, principalmente um para pessoas que moram sozinhas.

As informações sobre produtos conhecidas sobre o setor dos alimentos semiprontos demonstram uma demanda cada vez, além da preocupação com a qualidade e outras caracterizas destes produtos demonstrada pelos consumidores.

\subsection{Teste com Consumidores}

As características sensoriais da maior parte dos alimentos são essenciais para o sucesso mercadológico. Os testes de análise sensorial são ferramentas de extrema importância para a criação de novos produtos, já que mensuram opiniões de diversos consumidores, quanto à sua aceitação no mercado (SIDEL 1993 apud WALTER et al., 2010),

Assim é importante analisar a intenção de compra do consumidor, que "... é um processo decisório complexo, influenciado por vários fatores, incluindo preço, conveniência e marketing, sendo as características sensoriais determinantes na sua decisão" (GUERREIRO et al. (2000) apud WALTER, 2010).

Os testes sensoriais utilizam os sentidos humanos, como um meio de mensurar e devem ser incluídos como garantia da qualidade dos alimentos, por ser uma medida multidimensional integrada e por possuir muitos benefícios, como determinar a aceitação do produto pelos consumidores (CARDELLO et al. 1998).

\subsection{Broas de Milho}

Existem, segundo Moura (2012), diferentes tipos de broa de milho, tais como, por exemplo: branca; amarela, e; escura. A preferência por um tipo depende da tradição, ou seja, o consumidor terá tendência a gostar de um produto com características semelhantes as dos produtos que já conhece (MOURA, 2012).

ReLAInEP - Revista Latino-America de Inovação e Engenharia de Produção, Curitiba, PR, Brasil, v. 3, n. 4, p. 97-109, 2015. 


\section{ReLAInEP}

As Broinhas de Milho Saboreadas desenvolvidas, na pesquisa relatadas neste trabalho, tem textura semelhante maioria das broas de milho tradicionais, são crocantes, com um leve aroma de milho e feitas em tamanho pequeno com diâmetro de $5 \mathrm{~cm}$. O diferencial deste produto é o fato de ser recheado nos sabores goiabada, presunto e queijo ou frango (LERMEN et al., 2012). Uma foto ilustrativa, extraída de Lermen et al. (2012) das broas pode ser vista na Figura 1.

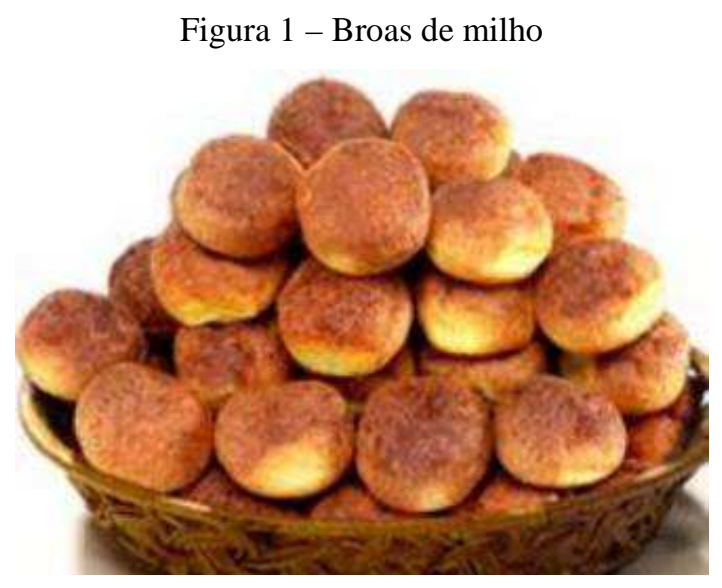

Como pode ser visto na Figura 1, as Broinhas de Milho Saboreadas têm cor um pouco diferente das broas tradicionais sendo um pouco mais escuras, devido a o tempo que elas necessitam no forno.

\section{REVISÃO DE LITERATURA}

$\mathrm{Na}$ revisão de literatura buscou-se utilizar trabalhos que estivessem inseridos nas áreas de Projeto do Produto e no Desenvolvimento de Novos Produtos, mas, sobretudo que mostrassem os procedimentos utilizados no desenvolvimento de novos produtos, dentro do foco do estudo, sendo: sabores; cores, e; aparência.

A pesquisa foi realizada nos portais Scielo e Capes, considerando artigos publicados a partir de 1999, no entanto, apenas 2 trabalhos, com este foco, nessas áreas específicas, foram encontrados.

Almeida, et. al. (1999), realizou um trabalho sobre a avaliação de figos em caldas, analisando o sabor, aroma e aparência. Ele conseguiu determinar que a variabilidade desses fatores nos figos em caldas pode ser ocasionada pela matéria-prima ou o meio de processamento inadequado.

ReLAInEP - Revista Latino-America de Inovação e Engenharia de Produção, Curitiba, PR, Brasil, v. 3, n. 4, p. 97-109, 2015. 


\section{ReLAInEP}

Cardoso et. al. (2010) desenvolveram um trabalho com o objetivo de estudar as oportunidades de um novo produto no mercado, por meio de pesquisas de mercado, para verificar se os consumidores iriam aceitar o novo produto. O novo produto conseguiu uma aprovação considerável de seus futuros consumidores, levando em conta alguns itens fundamentais de compra como preço, necessidade e ingredientes.

\section{METODOLOGIA}

Os métodos de abordagem adotados foram o qualitativo e o quantitativo. A pesquisa caracteriza-se quanto aos fins, como descritiva, exploratória e quanto aos meios bibliográfica, do tipo digital, e estudo de caso. A revisão de literatura foi realizada nos portais Scielo e Capes.

Foi realizado também, um Teste de Consumidores, no dia 31 de outubro de 2012, no período de 13h30min às 17h15min, nas dependências da Universidade Estadual do Paraná Campus de Campo Mourão. Neste Teste utilizou-se das técnicas de exposição e degustação do produto, sendo entrevistadas 64 pessoas, de maneira estruturada, por meio de um roteiro de entrevista. Com os dados obtidos, foi possível analisar o que os entrevistados acharam do produto, a necessidade de melhoria na fabricação do mesmo, para melhor atender a necessidade dos consumidores.

\section{RESULTADOS E DISCUSSÕES DO TESTE DE CONSUMIDORES}

No Teste de Consumidores, das 64 pessoas entrevistadas, $63 \%$ eram homens e $37 \%$ mulheres.

Além dos atributos sensoriais do produto (aparência, sabores e cores), vários outros aspectos das Broinhas de Milho Saboreadas foram analisados no Teste de Consumidores tais como valor, qualidade do produto e da embalagem.

Primeiramente, foi analisada a aparência do produto, os consumidores responderam o grau de qualidade a partir da aparência das Broinhas de Milho Saboreadas. Na Figura 2, apresenta-se os resultados da análise de aparência das Broinhas de Milho Saboreadas, obtidos com o Teste de Consumidores (LERMEN et al., 2012).

ReLAInEP - Revista Latino-America de Inovação e Engenharia de Produção, Curitiba, PR, Brasil, v. 3, n. 4, p. 97-109, 2015. 


\section{ReLAInEP}

Figura 2 - Análise da Aparência do Produto

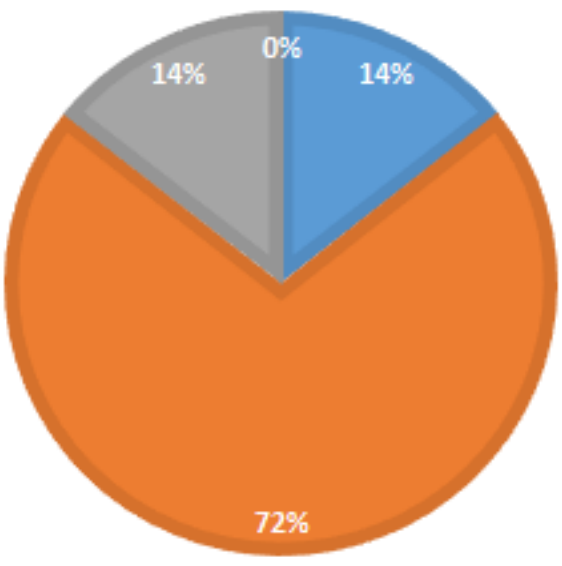

n Muito Atraente = Atraente = Pouco Atraente = Aparência Desagradável

Fonte: Os Autores

Das pessoas entrevistadas, 14\% acharam a aparência das Broinhas de Milho Saboreadas muito atraente, $72 \%$ atraente, $14 \%$ acharam a pouco atraente. Nenhum dos entrevistados achou que as Broinhas têm aparência desagradável. A partir destes resultados, é possível concluir que, a aparência das Broinhas de Milho Saboreadas teve uma ótima aceitação.

Em sequência, foi analisada a aceitação dos consumidores em relação a cor das Broinhas de Milho Saboreadas. Na Figura 3, apresenta-se os resultados desta análise, obtidos com o Teste de Consumidores (LERMEN et. al., 2012)..

Figura 3 - Análise da Cor do Produto

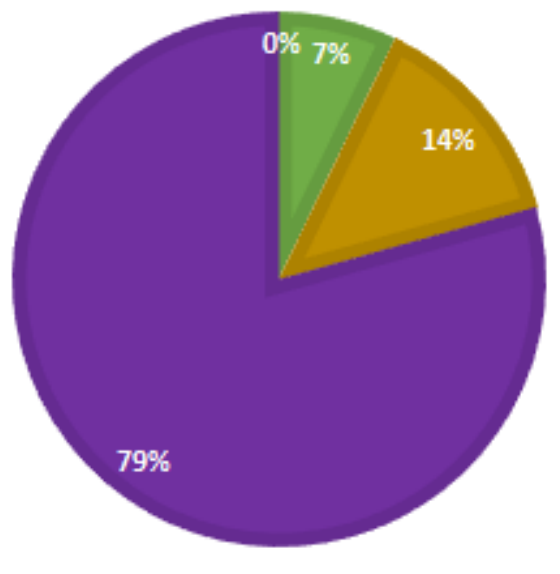

- Muito Bonita Bonita - Pouco Bonita a Cor Desagradável

Fonte: Os Autores

ReLAInEP - Revista Latino-America de Inovação e Engenharia de Produção, Curitiba, PR, Brasil, v. 3, n. 4, p. 97-109, 2015. 


\section{ReLAInEP}

Das pessoas entrevistadas, 7\% acharam a cor das Broinhas de Milho Saboreadas muito bonita, $14 \%$ acharam bonita, $79 \%$ acharam pouco bonita e nenhum dos entrevistados achou que a Broinha de milho tem a cor desagradável. Analisando estes resultados, foi possível concluir que, a cor das Broinhas de Milho Saboreadas teve uma aceitação baixa. Esta baixa aceitação ocorreu provavelmente devido a o fato de elas terem ficado muito tempo no forno. No entanto, este tempo de forno é necessário para garantir uma textura crocante.

Finalmente, foi analisada a aceitação dos consumidores em relação ao sabor das Broinhas de Milho Saboreadas. Na Figura 4, apresenta-se os resultados desta análise, obtidos com o Teste de Consumidores (LERMEN et. al., 2012).

Figura 4 - Análise do Sabor do Produto

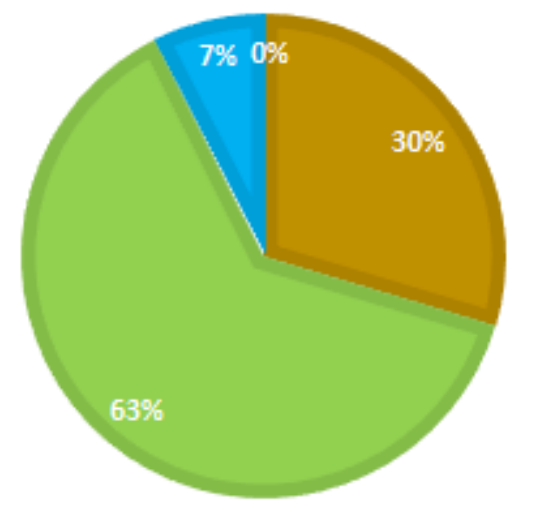

Fonte: Os Autores

Das pessoas entrevistadas, 30\% acharam o sabor das Broinhas de Milho Saboreadas muito saboroso, 63\% acharam saboroso, $7 \%$ acharam pouco saboroso e nenhum dos entrevistados achou que o produto tem um sabor desagradável. Avaliando estes resultados, é possível concluir que o sabor das Broinhas de Milho Saboreadas tivera uma ótima aceitação.

\section{CONCLUSÕES}

Observa-se que a inovação de produtos focados na área alimentícia vem sendo cada vez mais frequente, tendo em vista que os consumidores estão cada vez mais à procura de alimentos com praticidade.

As Broinhas de Milho Saboreadas são um exemplo evidente de praticidade e inovação, já que se enquadram na área de produtos congelados e panificação, tendo uma linha de quatro

ReLAInEP - Revista Latino-America de Inovação e Engenharia de Produção, Curitiba, PR, Brasil, v. 3, n. 4, p. 97-109, 2015. 
sabores. Com base neste artigo e nos artigos analisados para a conclusão deste, observou-se que o desenvolvimento de produtos focados na área alimentícia tornou-se maior.

Por meio do Teste de Consumidores foi possível observar que a aparência das Broinhas foi considerada atraente por $72 \%$ dos entrevistados, a cor foi considerada pouco bonita por $79 \%$ dos entrevistados e o sabor considerado saboroso por $63 \%$ dos entrevistados. Diante disso, pode-se observar que os pontos fortes das Broinhas de Milho Saboreadas são a aparência e o sabor, onde a cor deve ser aprimorada. Como recomendações aconselha-se a aplicação desta análise em outros projetos de novos produtos alimentícios, na área de panificação e congelados para forno.

\section{REFERENCIAS}

ALMEIDA, T.; FOLEGATTI, M.; FREIRE, M. T.; MADEIRA, M.; SILVA, F.; SILVA, M. A. Determinação do perfil sensorial e parâmetros de qualidade de figos em calda produzidos pela indústria brasileira. Ciência e Tecnologia de Alimentos. Vol. 19, No. 2. Campinas, 1999.

ASSOCIAÇÃO BRASILEIRA DE ENGENHARIA DE PRODUÇÃO (ABEPRO). Áreas e Subáreas de Engenharia de Produção. Rio de Janeiro, 2008.

BARBOSA, L. M. V.; FREITAS, R. J. S.; WASCSZYNSKYJ, N. Desenvolvimento de produtos e analise sensorial. Brasil Alimentos, São Paulo, v. 18, p 34-35, jan/fev. 2003.

BATTISTElla, N.; COLOMBO, J. R.; ABREU, K. C. K. Importância da Cor nas Embalagens como Fator Influenciador no Momento da Compra. Biblioteca online de Ciência da Comunicação. 2010. Disponível em: <http://www.bocc.ubi.pt/pag/bocc-kraemerembalagens.pdf > Acesso em 30 mai. 2013.

BAXTER, M. Projeto de produto: guia prático para o design de novos produtos. 2a ed. Editora Blucher: São Paulo, 2000.

CAMARGO, J. G. C. F.; QUEIRÓZ, T. R.; SCALCO, A. R. Estratégias de marketing na indústria de alimentos: o caso dos frigoríficos na região de Presidente Prudente-SP. In: XIV CONGRESSO DA SOCIEDADE BRASILEIRA DE ECONOMIA, ADMINISTRAÇÃO E SOCIOLOGIA RURAL. Anais... No. 14, 2007.

CANABRAVA, F.; BRUNET, J.; MESQUITA, M.; REIS, M. O Sentido da Comida: Será que só a fome é o tempero do alimento? ECLÉTICA. Anais... PUC - Rio. Jan/Jun, 2006.

CARDELlO, H. M. A. B.; CARDELlO, L. Teor de Vitamina C, Atividade de Ascorbato Oxidase e Perfil Sensorial da Manga (Mangífera índica L.) VAR, H. Durante o Amadurecimento. Ciência e Tecnologia de Alimentos. Campinas, v. 18, n. 2, maio, 1998.

ReLAInEP - Revista Latino-America de Inovação e Engenharia de Produção, Curitiba, PR, Brasil, v. 3, n. 4, p. 97-109, 2015. 
CARDOSO, W.; PINHEIRO, F.; PEREZ, R.; PATELLI, T.; FARIA, E. Desenvolvimento de uma salada de frutas: da pesquisa de mercado à tecnologia de alimentos. Ciência e Tecnologia de Alimentos. Vol. 30, No. 2. Campinas, 2010.

COBUCCI, R. M. A. Analise Sensorial: Apostila do Curso. Curso Tecnológico Superior em Gastronomia. Pontifica Universidade Católica de Goiás, PUC-GO, 2010.

GRUPO DE ENGENHARIA INTEGRADA - NÚCLEO DE MANUFATURA AVANÇADA (NUMA) - DEP - EESC - USP. Abordagem do Grupo de Engenharia Integrada para o Business Process Desenvolvimento de Produto. 2013.

LERMEN, F. H.; MODESTO, F. A.; LAURINO, L. N. A.; TORELI, L. A.; RODER, R. Projeto do Produto e o Desenvolvimento de um Novo Produto: Broinhas de milho Saboreadas (Chipas Paraguaias). Trabalho de Disciplina (Graduação em Engenharia de Produção Agroindustrial) - Departamento de Engenharia de Produção, Campus de Campo Mourão, Universidade Estadual do Paraná, UNESPAR, Campo Mourão/PR, 2012.

MATISTELLA, A. S.; MILAN, F. N. Estratégia de Marketing no segmento de produtos hortifrutícolas processados: Análise de uma empresa do setor. Revista de Ciências Gerais. Volume 8, No 17. 2009.

MOREIRA, D. A. Administração da Operação e Produções. 5 ed. São Paulo: Pioneira, 2000 .

MOURA, M. Qual a melhor broa de milho? Ciência Hoje -Jornal de Ciência, Tecnologia e Empreendedorismo. 19 de julho de 2012. Disponível em: <http://www.cienciahoje.pt/index.php?oid=54911\&op=all> Acesso: 27 set. 2013

PETER, J. P.; OLSON, J. Comportamento do Consumidor e Estratégia de Marketing. 8. ed. São Paulo: Mcgraw-Hill, 2009.

PROJECT MANAGMENTE INSTITUTE (PMI). PMI STANDARDS COMMITTEE. “A Guide to the Project Management Body of Knoledge”. p. 39-133. USA, 1996.

PROMON - Business \& Technology Review. Gerenciamento de Projetos. São Paulo, Brasil. 2008.

SEMENIK, R.; BAMOSSY, G. Princípios de Marketing: uma perspectiva global. São Paulo: Makron, 1995.

STRAPASSON, G.; LOPEZ, A. C.; BOSSO, T.; SANTOS, D.; MULINARI, R. A.; WILLI, G.; BARREIRA, S. Percepção de Sabor: Uma revisão. Visão Acadêmica, Curitiba, v. 12, p. 65-73, jan/jun. 2011.

VENTURA, R. Mudanças no perfil do Consumo no Brasil: Principais Tendências nos próximos 20 anos. Marcoplan - Prospectiva, Estratégia e Gestão. Ago 2010.

WALTER, E. H. M. et al. A influência de coberturas comestíveis na aceitação sensorial e intenção de compra de bolos de chocolate. Ciência e Tecnologia de Alimentos. Campinas, v. 30, n. 2, jun. 2010.

ReLAInEP - Revista Latino-America de Inovação e Engenharia de Produção, Curitiba, PR, Brasil, v. 3, n. 4, p. 97-109, 2015. 
ReLAInEP - Revista Latino-America de Inovação e Engenharia de Produção, Curitiba, PR, Brasil, v. 3, n. 4, p. 97-109, 2015. 